\title{
Revistas brasileiras publicadoras de artigos científicos em cirurgia. II - Terminologia e atribuições adotadas pelos editores. Proposta de organograma do periódico e fluxograma do artigo ${ }^{1}$
}

\author{
Dinah Aguiar Población \\ Saul Goldenberg ${ }^{3}$ \\ Edna Frasson de Souza Montero ${ }^{4}$ \\ Paulo de Oliveira Gomes \\ Heliane Campanatti-Ostiz ${ }^{6}$ \\ Márcia Bento Moreira ${ }^{7}$ \\ Aparecida Cássia dos $\operatorname{Santos}^{8}$ \\ Rosely de Fátima Pellizzon'
}

Población DA, Goldenberg S, Montero EFS, Gomes PO, Campanatti-Ostiz H, Moreira MB, Santos AC. Pellizzon RF. Revistas brasileiras publicadoras de artigos científicos em cirurgia. II - Terminologia e atribuições adotadas pelos editores: proposta de organograma do periódico e fluxograma do artigo. Acta Cir Bras [serial online] 2003 Nov-Dez; 18(6). Disponível em URL: http://www.scielo.br/acb.

RESUMO - Objetivo: Identificar a terminologia adotada pelo editor para designar os membros do corpo editorial e atribuições pertinentes aos componentes da equipe responsável pela política, administração e qualidade do conteúdo da revista. Propor organograma do periódico e fluxograma do artigo. Métodos: Foram analisados fascículos mais recentes de periódicos selecionados, disponíveis no Centro Latino-Americano e do Caribe de Informação em Ciências da Saúde e em várias bibliotecas médicas do Brasil, apresentados na primeira parte desta linha de pesquisa. Nesta amostragem identificou-se a terminologia e as atribuições adotadas pelos editores de cada revista para designar os responsáveis pela forma e conteúdo do periódico. Resultados: A terminologia adotada para o corpo editorial constante de cada revista foi levantada mostrando a diversificação de termos adotados para funções semelhantes. Foram identificados 74 termos, designando atividades exercidas pelos membros do corpo editorial, envolvidos com a produção e difusão da revista, e agrupados em 5 categorias. Visto sob a perspectiva de editoração, quer impressa ou eletrônica, a categorização das diferentes etapas do processo proporcionou os subsídios necessários para a elaboração do "Organograma da Revista Científica". A interpretação das atividades e responsabilidades de cada membro no organograma está descrita no "Perfil e responsabilidades de membro do corpo editorial". Ao inserir cada membro do corpo editorial, de acordo com as respectivas responsabilidades, foi possível elaborar o "Fluxograma do artigo". Conclusão: Não há uniformidade de termos e atribuições para a designação dos membros do corpo editorial nos periódicos estudados. Propõe-se organograma do periódico e fluxograma do artigo.

DESCRITORES - Terminologia [tipo de publicação] Editoração. Publicações periódicas. Comunicação científica. Produção científica.

1 Trabalho do Núcleo de Comunicação Científica em Cirurgia (NCCC) da Sociedade Brasileira para o Desenvolvimento da Pesquisa em Cirurgia (SOBRADPEC).

2 Profa. Dra. da Escola de Comunicação e Artes (ECA-USP). Coordenadora do Núcleo de Pesquisa de Produção Científica em Ciência da Informação (NPC-ECA-USP) e do Núcleo de Comunicação Científica em Cirurgia (NCCC) da SOBRADPEC. dinahmap@usp.br

3 Prof. Titular (Aposentado) do Depto. de Cirurgia da UNIFESP-EPM. Presidente da SOBRADPEC. sgolden@terra.com.br

4 Professora Afiliada do Depto. de Cirurgia da UNIFESP-EPM e Pesquisadora do NCCC. efsmontero.dcir@epm.br

5 Prof. Adjunto do Depto de Cirurgia da UNIFESP-EPM e Pesquisador do NCCC. pgomes.dcir@epm.br

6 Editora executiva da Pro-Fono Revista de Atualização Científica, membro do NCCC, Pós-Graduanda da FFLCH - USP. campanattiostiz@profono.com.br

7 Médica Veterinária, Mestre em Técnica Operatória e Cirurgia Experimental e membro do NCCC. mazinhabm.dcir@epm.br

8 Médica Veterinária, Pós-Graduanda da Radiologia Clínica da UNIFESP-EPM e membro do NCCC. cassiast@bol.com.br

9 Bibliotecária responsável pelo setor de referência da Biblioteca Central da UNIFESP-EPM e membro do NCCC. rosely.bc@epm.br 


\section{Introdução}

A atividade científica e tecnológica, que se desenvolve primordialmente nas universidades e institutos de pesquisa, é estimulada e apoiada pelas sociedades científicas que atuam como parceiras, investindo na difusão do conhecimento produzido. As sociedades científicas têm como missão congregar especialistas, promover eventos e publicações onde transparece a força e a competência de uma comunidade que se impõe como geradora de conhecimentos. Os objetivos a serem alcançados pelas sociedades científicas são semelhantes, quer em áreas similares, quer situadas em diferentes países.

Essas atividades, iniciadas há quatro séculos, quando as primeiras sociedades científicas mostravam preocupação com o controle do "excessivo número de publicações e de cartas científicas" conduziram ao início do processo de controle de qualidade desde 1665, quando foi criada a Royal Society of London, ao autorizar a primeira publicação científica "Philosophical Transactions of the Royal Society of London" com a exigência do crivo de avaliação de alguns membros da sociedade ${ }^{1}$. Segundo Price $(1963)^{2}$ é surpreendente que "o mundo científico atual não se diferencia em absoluto do existente no século XVII". Assim, confirma que a ciência continua sendo sempre moderna e que a maioria dos conhecimentos consta da biografia dos autores que ainda estão vivos e atuantes. Com essa visão, relacionando ciência e sociedade, Price ${ }^{2}$ identifica o rápido crescimento atual da ciência e aplica o método científico para investigar a função das publicações e a evolução das organizações que reuniam os cientistas, cujo nome foi usado pela primeira vez em 1833, numa reunião da British Association for the Advancement of Science ${ }^{1}$.

Nas décadas de 50 e 60 do século $\mathrm{XX}$, a comunidade científica descoberta há pouco mais de um século como tal, era estudada por vários sociólogos que procuravam entender a sua dinâmica interna, segundo várias tendências. A partir de 1970, os diversos tipos de atividades científicas despertaram maior interesse dos sociólogos da ciência que procuravam respostas para questões consideradas relevantes na década de 60 .
A dinâmica da ciência divulgada pelas revistas, que foram sendo publicadas nas primeiras décadas, começou a ser objeto de análise de editores que sentiam a necessidade de estruturar as publicações segundo algumas diretrizes que posteriormente se transformaram em recomendações e normas. Na década de 70 do século XX a União das Nações Unidas para a Educação, a Ciência e a Cultura (UNESCO) convidou o editor de uma revista de química para elaborar o "Guidelines for Editors of Scientific and Technical Journals" 3, no qual são descritas as primeiras funções do editor científico, a estrutura dos periódicos e a forma de apresentação de artigos científicos, destacando a principal habilidade do editor para atingir o leitor mediante os recursos dos processos de recuperação da informação.

Com preocupações semelhantes, destaca-se a relevância da linha de pesquisa, que está sendo desenvolvida pelo Núcleo de Comunicação Científica em Cirurgia $(\mathrm{NCCC})^{4}$ em parceria com o Núcleo de Produção Científica (NPC) da ECA - USP, refletindo a dinâmica da comunidade de pesquisadores brasileiros e do "Colégio Virtual" dessa área específica, que é representada pelos autores, editores, "peer review" e pelas sociedades científicas que editam as revistas. A importância do papel destes atores é confirmada durante as entrevistas e contatos que vêm sendo feitos com editores das revistas médicas a partir do acesso ao artigo publicado na Acta Cirúrgica Brasileira em dezembro de $2002^{5}$.

O objetivo proposto para a segunda etapa desta linha de pesquisa é identificar a terminologia adotada pelo editor para designar os membros do corpo editorial e atribuições pertinentes aos componentes da equipe responsável pela política, administração e qualidade do conteúdo da revista. Propor organograma do periódico e fluxograma do artigo.

\section{Métodos}

Os fascículos mais recentes disponíveis na BIREME e em várias bibliotecas médicas do país, dos periódicos selecionados que constituíram o universo da pesquisa, descrito na primeira parte desta linha de pesquisa ${ }^{5}$ foram analisados visando identificar a terminologia adotada pelos editores de cada revista para designar os responsáveis pelo conteúdo e forma do periódico.

- A terminologia coletada foi organizada em ordem alfabética e categorizada de acordo com as funções básicas do corpo editorial.

- Realização de entrevista semiestruturada com os editores, que receberam com antecedência a lista dos termos em ordem alfabética e com categorização preliminar.

- Identificação da terminologia em fontes especializadas: dicionários técni$\cos ^{6-9}$, glossários ${ }^{10,11}$, definições dadas em cursos de editoração científica ${ }^{12}$, vocabulário técnico ${ }^{13}$ e capítulos de livros de manuais de estilo ${ }^{14-15}$.

- Apresentação dos resultados parciais na I Reunião dos Editores de Revistas Médicas Publicadoras de Contribuições Clínico-Cirúrgicas, realizada na sede da Associação Médica Brasileira em São Paulo em 23 de abril de 2003, patrocinada pela SOBRADPEC. Cada editor foi convidado a contribuir para o aprimoramento da terminologia e das respectivas conceituações e atribuições dos membros do Corpo Editorial das suas respectivas revistas;

- Elaboração do Organograma da Revista; do Perfil e Responsabilidade de Cada Membro do Corpo Editorial; e estruturação do Fluxograma do Artigo.

- Submissão da proposta aos editores presentes à II Reunião dos Editores de Revistas Médicas Publicadoras de Contribuições Clínico-Cirúrgicas, realizada em São Paulo em 15 de outubro de 2003. Após as discussões foram solicitadas aos participantes as sugestões e contribuições para operacionalizar as atividades dos editores das revistas.

\section{Resultados}

A terminologia adotada para o corpo editorial constante de cada revista foi levantada mostrando a diversificação de termos adotados para funções semelhantes. Com o objetivo de facilitar a compreensão das atividades exercidas pelos membros do corpo de profissionais envolvidos com a produção e difusão da revista, os 74 termos foram agrupados em categorias (Quadro 1). 
O Quadro 1, enviado antecipadamente aos editores das revistas que fazem parte do universo, estava inserido no roteiro da entrevista semi-estruturada e contribuiu para a análise. As discussões sobre as posições dos editores ocorreram durante a I Reunião dos Editores de Revistas Médicas Publicadoras de Contribuições Clínico-Cirúrgicas. Os termos apresentados alfabeticamente comprovaram a dispersão terminológica para atividades semelhantes. Por outro lado, a categorização permite identificar as diferentes etapas dos processos desde o recebimento do artigo, da administração das atividades de protocolo e correspondência com o autor e com o encaminhamento para as etapas da avaliação científica do artigo, desde a estrutura da forma até o conteúdo científico, com as possibilidades de aceitação, rejeição e devolução. Visto sob a perspectiva de editoração, quer impressa ou eletrônica, a categorização das diferentes etapas do processo facilitou os subsídios necessários para a elaboração do Organograma da Revista Científica (Figura 1).

A interpretação das atividades e responsabilidades de cada membro no Organograma estão descritas no "Perfil e responsabilidades de membro do corpo editorial" (Quadro 2).
QUADRO 1 - Lista categorizada dos termos adotados pelas revistas avaliadas.

\begin{tabular}{|c|c|c|c|}
\hline $\begin{array}{l}\quad \text { Responsabilidac } \\
\text { Co-editor } \\
\text { Diretor } \\
\text { Diretor científico } \\
\text { Diretor científico adjunto } \\
\text { Diretor responsável } \\
\text { Diretoria } \\
\text { Editor (a) / Fundador } \\
\text { Editor-chefe } \\
\text { Editor adjunto } \\
\text { Editor(es) anterior(es) Edit } \\
\text { Editor assistente } \\
\text { Editor (es) -responsável (ei } \\
\text { Editor científico } \\
\text { Editor científico responsáv } \\
\text { Editor emérito } \\
\text { Editor executivo } \\
\text { Editor (es) titular (es)Edito }\end{array}$ & $\begin{array}{l}\text { tor(es) associado(s) } \\
\text { is) / Responsável } \\
\text { vel } \\
\text { ria executiva }\end{array}$ & $\begin{array}{l}\quad \text { Anál } \\
\text { Avaliador } \\
\text { Comissão } \\
\text { Conselho } \\
\text { Conselho } \\
\text { Conselho } \\
\text { Conselho } \\
\text { Conselho } \\
\text { Conselho } \\
\text { Consulto } \\
\text { Consulto } \\
\text { Coordena } \\
\text { Corpo ed } \\
\text { Corpo ed } \\
\text { Corpo ed } \\
\text { Corpo de }\end{array}$ & $\begin{array}{l}\text { se de Conteúdo Científico } \\
\text { es } \\
\text { editorial } \\
\text { científico } \\
\text { consultivo } \\
\text { consultores nacional } \\
\text { consultores internacional } \\
\text { deliberativo } \\
\text { editorialConsultor editorial } \\
\text { es } \\
\text { es externos } \\
\text { dor } \\
\text { torial } \\
\text { torial internacional } \\
\text { torial nacional } \\
\text { revisores }\end{array}$ \\
\hline $\begin{array}{l}\text { Normalização } \\
\text { Comissão de redação } \\
\text { Comissão de normalização } \\
\text { Editoração em inglês } \\
\text { Produção e normalização } \\
\text { Redator chefe } \\
\text { Redator secretário } \\
\text { Redatores } \\
\text { Revisão } \\
\text { Revisão em inglês }\end{array}$ & $\begin{array}{l}\text { Apoio Técnico } \\
\text { Assessoria técnica } \\
\text { Capa } \\
\text { Comissão executiv } \\
\text { Conselho administ } \\
\text { Diretor administra } \\
\text { Editoração para in } \\
\text { Editoração Eletrôn } \\
\text { Impressão e acabaı } \\
\text { Produção editorial } \\
\text { Produção gráfica } \\
\text { Projeto gráfico e d } \\
\text { Secretaria executiv } \\
\text { Secretário } \\
\text { Tesoureiro }\end{array}$ & $\begin{array}{l} \\
\text { ivo } \\
\text { o } \\
\text { a } \\
\text { nto } \\
\text { gramação }\end{array}$ & $\begin{array}{l}\text { Comunicação e Marketing } \\
\text { Comunicação científica } \\
\text { Diretoria de Comunicação } \\
\text { Diretor de Publicações } \\
\text { Assessoria } \\
\text { Assessoria de comunicação } \\
\text { Assessoria de difusão } \\
\text { Assessoria de divulgação } \\
\text { Secretaria de divulgação } \\
\text { Assessoria de publicidade } \\
\text { Assistente de publicidade } \\
\text { Jornalista } \\
\text { Jornalista responsável } \\
\text { Publicidade } \\
\text { Gerência comercial } \\
\text { Gerente comercial }\end{array}$ \\
\hline
\end{tabular}

QUADRO 2 - Perfil e responsabilidade de cada membro do Corpo Editorial.

Texto para apreciação, discussão e aperfeiçoamento, de uma proposta básica de composição do Corpo Editorial, facilitando a identificação e a delimitação de responsabilidade e funções.

Fundador: cientista de renome, responsável pela criação da revista, independentemente do período de atuação.

Editor Científico: pesquisador, de alto nível, responsável pela execução da Política Editorial e pelo conteúdo científico do periódico. Ocupa a posição mais elevada na hierarquia do organograma do Corpo Editorial da revista.

Editor Consultivo: profissional de larga experiência, com conhecimentos de editoração e domínio da comunicação científica, a quem o Editor Científico recorre para se aconselhar e discutir as suas dúvidas e as questões relativas à visibilidade e ao impacto da publicação.

Secretária Executiva: é o braço direito do Editor Científico. Profissional competente e dinâmica que interage com praticamente todos os membros do Corpo Editorial. Compartilha a supervisão da rotina.

Editores Assistentes: pesquisadores que coordenam as atividades relacionadas com o conteúdo e forma dos artigos e continuamente interrelacionam com o editor científico. Conteúdo: atua diretamente na supervisão do conteúdo científico dos artigos. Forma: atua diretamente na supervisão da forma, da normalização dos artigos, da produção editorial e do marketing. Interage com os Editores Científico e Consultivo.

Editores Associados: Especialista da área e/ou pesquisador que participa freqüente e intensivamente da preparação científica de originais nas áreas de sua especialidade (encomendando e encaminhando contribuições, designando árbitros, analisando pareceres, etc.). Podendo ser denominado de Editor de Seção quando responsável por áreas específicas. Editor Convidado: constará da publicação quando for designado para coordenar e dirigir a elaboração de um fascículo, número ou suplemento específicos.

Pareceristas: médicos/pesquisadores designados para analisar artigos de sua competência.

Consultores Nacionais e Internacionais: pesquisadores com destaque nacional e internacional na sua área de atuação que colaboram técnica e cientificamente, conferindo credibilidade ao periódico.

Normalização e Nomenclatura: profissionais especializados na padronização das citações e referências dos artigos, correções dos idiomas e da nomenclatura científica.

Produção Editorial: profissionais especializados na preparação do periódico para o formato eletrônico e impressão gráfica.

Marketing: profissionais que dominam os processos de difusão e de divulgação científica, assim como da identificação de fontes financiadoras e estratégias de captação de recursos. 
Ao inserir cada membro do corpo editorial, de acordo com as respectivas responsabilidades, foi possível elaborar o Fluxograma do artigo (Figura 2), que permite acompanhar desde o início do recebimento do artigo enviado pelo autor, de acordo com as "instruções aos autores" constantes da revista, até o processo de aprovação ou rejeição do documento.

O organograma da revista científica (Figura 1), o perfil e responsabilidade de cada membro do corpo editorial (Quadro 2) e o fluxograma do artigo (Figura 2) foram enviados antecipadamente aos editores que, durante a II Reunião dos Editores de Revistas Médicas Publicadoras de Contribuições Clínico-Cirúrgicas, interpretaram a proposta apresentada pelo NCCC, resultando em ampla discussão. Os editores participantes desta II Reunião foram convidados a refletir sobre a contribuição do NCCC, que representa uma versão atualizada de diretrizes constantes de trabalhos anteriores ${ }^{3}$ que poderá ser adotada com o suporte de novas tecnologias que certamente serão privilegiadas no processo da editoração das Revistas Eletrônicas.

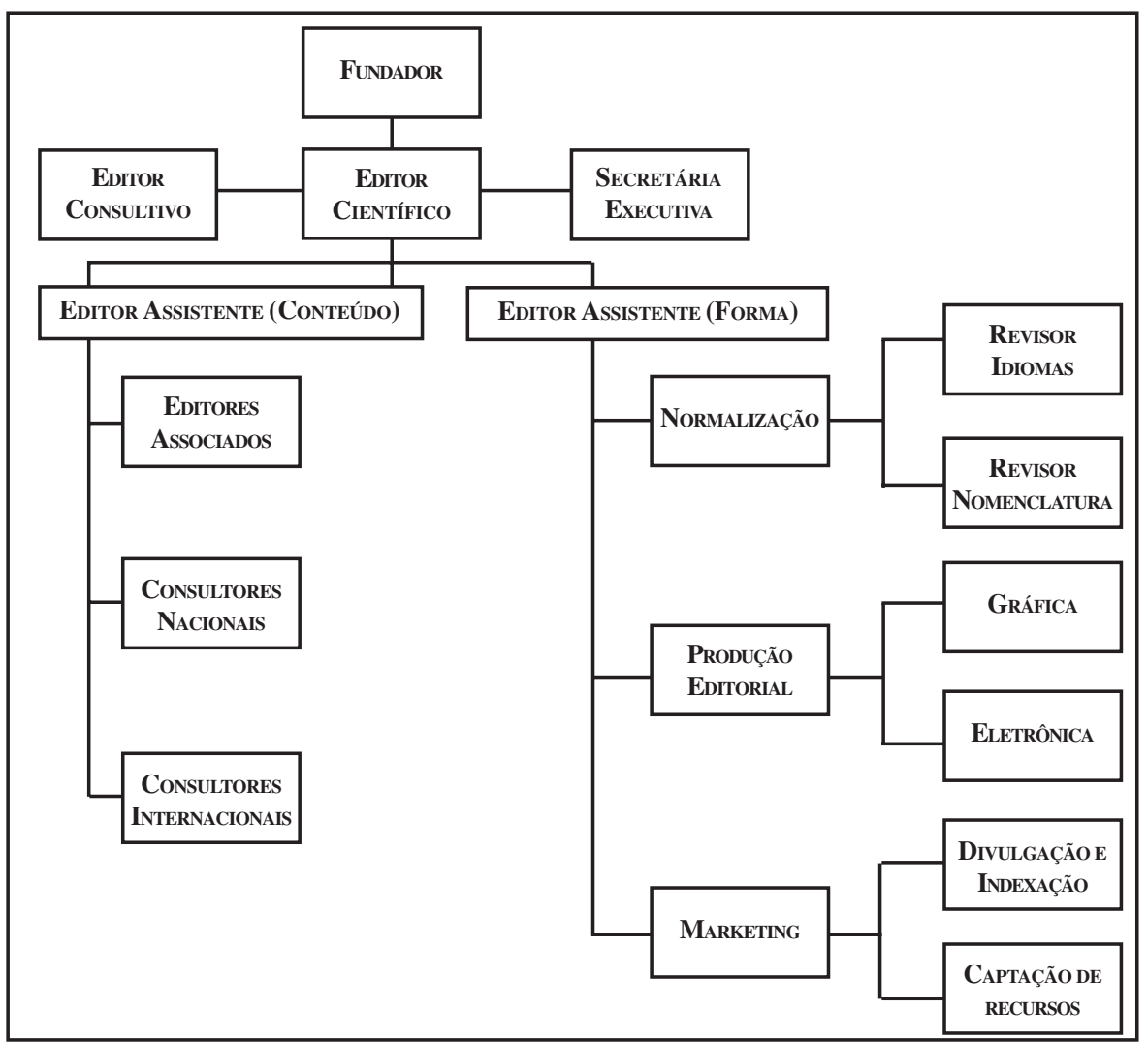

FIGURA 1 - Organograma da revista científica.

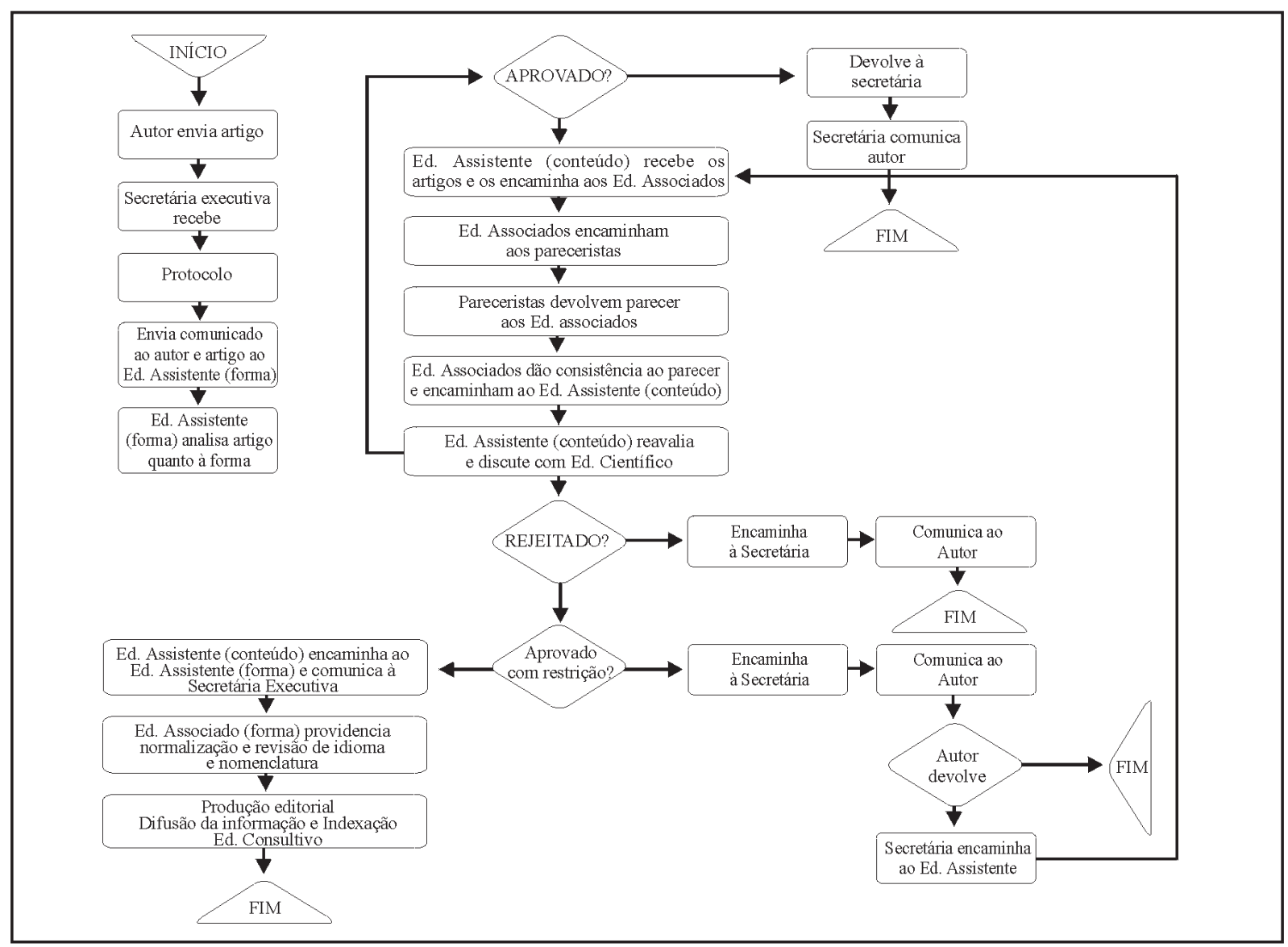

FIGURA 2 - Fluxograma do artigo. 


\section{Discussão}

A pesquisa realizada pelo NCCC submetida à discussão dos dados com os Editores das Revistas que fazem parte do universo mostrou que é viável atingir o consenso. $\mathrm{O}$ interesse comprovado pela contribuição dos editores que enviaram propostas simplificadas, em que atividades similares foram apresentadas contemplando as mesmas etapas, representaram formas diferentes, sem o detalhamento técnico das estruturas organizacionais de fluxograma e organograma. A expectativa é que brevemente seja alcançado o padrão de qualidade desejável, que permita não só a visibilidade da produção científica nacional, mas que atinja o fator de impacto compatível com os demais periódicos internacionais da área.

A quantidade de 74 termos adotados atualmente pelos editores poderá ser reduzida a 11 termos (com algumas especificações), que não implicam necessariamente na alocação de pessoas diferentes, embora as funções sejam exercidas de acordo com o fluxograma apresentado.

As próximas etapas desta linha de pesquisa contemplarão a seqüência proposta na parte $\mathrm{I}^{5}$ dos artigos programados nesta série e analisarão os autores quanto à observância das instruções aos autores complementando posteriormente com o perfil que será identificado para compreender a competitividade da produção nacional em relação à internacional, que influencia na determinação do fator de impacto.

\section{Conclusão}

Não há uniformidade de termos e atribuições para a designação dos membros do corpo editorial nos periódicos estudados. Propõe-se organograma do periódico e fluxograma do artigo.

\section{Referências}

1. Velho L. Indicadores científicos: em busca de uma teoria. Interciência 1990;15(3):139-45.

2. Price DS. Little science, big science. New York: Columbia Univ. Press; 1963.

3. Grünewald H. Guidelines for editors of scientific and technical journals. Paris: UNESCO/INISIST, 1979. (DGI - 79/WS/8)

4. Goldenberg S. Núcleo de comunicação científica em cirurgia (NCCC) e núcleo dos amigos da cirurgia experimental (NACE) da SOBRADPEC. Acta Cir Bras 2002;17(2):87-8

5. Población DA, Goldenberg S, Montero EFS, Moreira MB, Pellizzon RF. Revistas brasileiras publicadoras de artigos científicos em cirurgia. I - Características estruturais e administrativas das revistas. Acta Cir Bras 2002;17(6):359-69.

6. Hollanda ABF. Novo dicionário básico da língua portuguesa. Rio de Janeiro: Editora Nova Fronteira; 1995.

7. Claudin V, Anabitarte H. Diccionario general de la comunicaión. Barcelona: Mitre; 1986.

8. Gil BM, Trautman R, Goy P. Diccionario técnico de biblioteconomia: español-inglês:
Technical dictionary of librarianship: english-spanish. México: Trilhas; 1973.

9. Weiner R. Webster's new world dictionary of media and communications. New York: Webster's New World; 1990.

10. Thompson EH. A.L.A. Glossary of library terms with a selection of terms in related fields. Chicago: A.L.A.; 1975

11. Young $\mathrm{H}$ (Ed). Glosario ALA de bibliotecología y ciencias de la información. Madrid: Diaz de Santos; 1988

12. Trzesniak P. Cargos e funções associados à publicação científica: uma proposta de nomenclatura. In: Curso de editoração científica, 10, Atibaia, 2001. Resumos. Atibaia: ABEC 2001.

13. Ferreira IG, Furgler NBM. Mídia: uma tentativa de padronização do vocabulário técnico de mídia na língua corrente do país. SLP: Edições Mercado Global; 1977. v.1.

14. American Medical Association. Iverson C. Manual of style: a guide for authors and editors. Philadelphia: Williamns \& Wilkins; 1998.

15. Youdeowel A. Editorial responsibilities. In A guidebook on journal publishing for agriculture and rural development. Oxford: INASP; 2001.

\section{Agradecimentos}

Ao Prof. Dr. Waldomiro C. S. Vergueiro, Chefe do Departamento de Biblioteconomia e Documentação da ECA - USP, pela colaboração na estruturação do fluxograma do artigo.

A Profa. Dra. Regina C. Figueiredo Castro, Vice-Diretora da BIREME, pela colaboração nas discussões relativas ao tema.

Población DA, Goldenberg S, Montero EFS, Gomes PO, Campanatti-Ostiz H, Moreira MB, Santos AC, Pellizzon RF. Brazilian journal publisher of scientific papers in surgery. II - Terminology and attributions adopted by the editors: proposition of journal organogram and article fluxogram. Acta Cir Bras [serial online] 2003 Nov-Dec; 18(6). Available from URL: http://www.scielo.br/acb. ABSTRACT - Purpose: To identify the terminology adopted by the editor to assign the editorial board members and attributions related to the team components responsible for the policies, administration, and contents quality of the journal. Methods: It was analyzed more recent numbers from selected journals, available at Latin American and Caribbean Center on Health Sciences Information and other medical libraries from Brazil, presented at the first part of this research. In this sample, it was identified the terminology and attributions adopted by the editors from each journal to designate the responsible for the content and the style of the journal. To recommend the journal organogram and the article fluxogram. Results: The adopted terminology for the editorial board in each journal was collected showing the diversification of the terms for similar functions. It was identified 74 terms, classified in five categories, assigning activities exerted by the editorial board members, involved in the journal production and diffusion. Under the editorial perspective, printed or electronic, the categorization of the different phases of the process provided subsidies to elaborate the "Organogram of the scientific periodical". The interpretation of the activities and responsibilities of each member in the organogram is described in the "Profile and responsibilities of the editorial board members". To insert each member of the editorial board, according to its respective responsibility, it was possible to elaborate the "Paper fluxogram". Conclusion: There is no uniformity to designate terms and attributions for the members of the editorial board in the studied journals. Recommendations are presented for the journal organogram and the article fluxogram.

KEY WORDS - Terminology [publication type]. Publishing. Periodicals. Scientific communication. Scientific production.

Correspondência:

Profa. Dinah Aguiar Población

Rua Jorge Rizzo, 217/133

05424-060 São Paulo - SP

Tel/Fax: (11)3815-7309
Conflito de interesse: nenhum Fonte de financiamento: nenhuma

Data do recebimento: 19/11/2003

Data da revisão: $24 / 11 / 2003$

Data da aprovação: 25/11/2003 